\title{
Predictive value of C-reactive protein after drug-eluting stent implantation: an update view
}

\author{
Rocco A Montone ${ }^{1}$ \& Giampaolo Niccoli*,1 \\ ${ }^{1}$ Department of Cardiovascular \& Thoracic Sciences, Fondazione Policlinico Universitario A. Gemelli IRCCS, Rome, Italy; Università \\ Cattolica del Sacro Cuore, Rome, Italy. \\ *Author for correspondence: Tel.: +39 (0)2 30154187; gniccoli73@hotmail.it
}

\author{
"Evidence deriving from clinical studies suggests that both short-term and long-term clinical \\ outcome of patients undergoing $\mathrm{PCl}$ is clearly associated with the inflammatory status before and \\ after stent implantation"
}

First draft submitted: 26 March 2018; Accepted for publication: 25 July 2018; Published online: 20 September 2018

Keywords: coronary artery disease $\bullet$ C-reactive protein $\bullet$ drug-eluting stent $\bullet$ in-stent restenosis $\bullet$ percutaneous coronary intervention

Coronary artery disease represents the leading cause of morbidity and mortality in the western countries and percutaneous coronary intervention (PCI) with drug-eluting stent (DES) implantation is the most widely performed procedure for the treatment of symptomatic coronary artery disease [1].

Previous studies clearly showed that PCI may induce a vessel wall response characterized by an inflammatory response that is involved in the pathogeneses of in-stent restenosis (ISR) and stent thrombosis (ST) [2-5]. Drug-eluting stents were designed to reduce in-stent neointimal proliferation and minimize the occurrence of ISR [1,2]. However, several studies demonstrated that ISR still occurred after first-generation DES implantation [1-5]. Moreover, concern had been raised regarding the risk of late (between 30 days and 1 year) and very late (>1-year) ST [2] and the occurrence of in-stent neoatherosclerosis after first-generation DES implantation [2]. Of importance, data deriving studies evaluating second-generation DES showed significantly improved clinical and angiographic outcomes compared with first-generation DES, as a consequence of technical improvements in strut design, drug-delivery and polymer coating [1].

Several studies suggested a critical role for inflammatory reaction in the pathogenesis of adverse events after DES implantation and different inflammatory biomarkers had been studied in order to stratify prognosis in patients undergoing DES implantation. In particular, C-reactive protein (CRP) represents the most extensively studied inflammatory biomarker in the cardiovascular field [3].

CRP is an acute-phase protein that is synthesized upon stimulation by inflammatory cytokines (mainly IL6) and is secreted into the circulation [3]. CRP represents a sensitive marker of systemic inflammation and has been demonstrated to predict the occurrence of adverse cardiovascular events both in primary and secondary prevention [3]. Moreover, CRP is useful to predict both clinical and angiographic outcomes in patients undergoing bare-metal stent (BMS) implantation [3].

Of importance, CRP has been extensively investigated in the setting of PCI with first-generation DES. In particular, several studies evaluated the prognostic role of both baseline and postprocedural CRP levels.

\section{Baseline CRP levels \& prognosis after DES implantation}

Prognostic value of baseline CRP levels was first assessed in a study by Park et al. [6] (2691 patients, median followup 3.9 years), demonstrating a clear relationship between CRP and DES thrombosis. Subsequent studies with a medium- to long-term follow-up confirmed the prognostic role of baseline CRP for thrombotic events after DES implantation [7]. A recent study by Oemrawsingh et al. [8] evaluated long-term prognostic value of baseline CRP in PCI with sirolimus-eluting stent demonstrating that higher CRP levels were associated with a higher incidence 
of all-cause mortality or myocardial infarction (MI) at 10-year follow-up also after adjustment for established cardiovascular risk factors.

An interesting study by Schoos et al. [9] evaluated the prognostic value of baseline hs-CRP in relation to type of stent implanted in 301 patients with ST-segment elevation myocardial infarction. Patients were categorized based on stent-type and hs-CRP levels. Hs-CRP $>2 \mathrm{mg} / \mathrm{l}$ (HR: 2.7; 95\% CI: 1.3-5.6; $\mathrm{p}=0.007$ ) and the combined variable of hs-CRP $>2 \mathrm{mg} / \mathrm{l}$ and BMS (HR 2.4; 95\% CI: $1.2-4.5 ; \mathrm{p}=0.006$ ) independently predicted the composite end point of death and MI at 36-month follow-up. The interaction between hs-CRP and stent type was significant $(\mathrm{p}=0.0006)$. Survival analysis demonstrated significant differences for occurrence of death and MI: $4.8 \%$ in BMS + CRP $\leq 2 \mathrm{mg} / 1,11.9 \%$ in DES + CRP $\leq 2 \mathrm{mg} / 1,17.6 \%$ in DES + CRP $>2 \mathrm{mg} / \mathrm{l}$, and $27.9 \%$ in BMS + CRP $>2 \mathrm{mg} / \mathrm{l}$. Of the 14 stent thromboses, none occurred in patients with BMS $+\mathrm{CRP} \leq 2 \mathrm{mg} / \mathrm{l}$. In particular, these data seem to suggest that BMS implantation should be favored when hs-CRP is $\leq 2 \mathrm{mg} / \mathrm{l}$ and DES when hs-CRP is $>2 \mathrm{mg} / \mathrm{l}$ in order to reduce long-term adverse outcomes including stent thrombosis in ST-segment elevation myocardial infarction patients treated with primary PCI.

Moreover, a recent study by Niccoli et al. [10] demonstrated that in patients undergoing DES implantation in bifurcation lesions, higher baseline CRP levels are associated with a higher incidence and extent of periprocedural MI, that in turn may have prognostic implications.

Finally, another study by Niccoli et al. [11], enrolling patients presenting with ISR after DES, demonstrated that baseline CRP levels at the time of the first procedure are associated with a more aggressive (diffuse) ISR pattern.

\section{Postprocedural CRP change \& prognosis after DES implantation}

Several studies have addressed the change in CRP levels from baseline after DES implantation and investigated whether this change might affect the outcome.

A recent study by Shiba et al. [12] enrolled 1234 consecutive patients undergoing DES ( $>60 \%$ first-generation DES) implantation and CRP was measured both at baseline and 8-12 months (late phase) after stenting. This study showed that high levels of CRP ( $>2 \mathrm{mg} / \mathrm{dl}$ ) was detected in $38.0 \%$ of patients at baseline and in $23.6 \%$ during late phase, with an increased risk of major adverse cardiac events (MACE) (composite of death, nonfatal MI, recurrence of revascularization) for patients with CRP levels $>2.0 \mathrm{mg} / \mathrm{l}$ at baseline (HR: 1.52; 95\% CI: 1.21-1.93; $\mathrm{p}=0.0004)$ and in the late phase (HR: 4.00; 95\% CI: 3.16-5.05; $\mathrm{p}<0.0001)$. Of interest, this study showed that, as compared with baseline CRP elevation, late-phase CRP elevation was a more powerful predictor of MACE after DES implantation. Moreover, about two-thirds of the patients with late-phase CRP elevation also had high baseline CRP levels, suggesting the presence of a chronic inflammation that persists after revascularization. Of note, at multivariate analysis newer-generation DES (OR: 0.59; 95\% CI: 0.41-0.84; $\mathrm{p}=0.003$ ) and statin therapy (OR: 0.68; 95\% CI: $0.47-0.97 ; \mathrm{p}=0.03$ ) were negatively associated with late-phase CRP elevation.

Accordingly, a recent study by Hsieh et al. [13] evaluating the prognostic value of CRP levels measured at 9-month after PCI with DES. A total of 1763 patients who received 9-month follow-up angiography were enrolled and grouped according to hs-CRP level 9 months after the DES implantation. Of importance, patients with CRP $>3 \mathrm{mg} / \mathrm{dl}$ had a lower cardiovascular event-free survival rate than patients with CRP $<3 \mathrm{mg} / \mathrm{dl}$. Multivariate analysis showed that a follow-up hsCRP level $>3.0 \mathrm{mg} / \mathrm{l}$ was an independent predictor of a MACE (cardiac death, reinfarction, target lesion revascularization and stenting in a new lesion, or coronary bypass surgery).

Kang et al. [14] investigated the relationship between inflammatory markers and neointimal hyperplasia (NIH) after DES implantation. They enrolled 42 consecutive patients undergoing PCI with a single DES for stable angina and single de novo stenosis. Inflammatory biomarkers were measured before, at 24 and $72 \mathrm{~h}$ after PCI. Follow-up angiographic and IVUS examinations were performed 9 months after index procedure. No relationship was noted between the baseline hs-CRP level and NIH. However, a significant positive correlation was noted between NIH and the hs-CRP level obtained at 24 and $72 \mathrm{~h}$ after the procedure, with a positive correlation between the change in the hs-CRP level and NIH at $24 \mathrm{~h}(\mathrm{r}=0.414, \mathrm{p}=0.006)$. Similar findings were found by Lee et al. in an optical coherence tomography follow-up study [15].

\section{Conclusion}

Evidence deriving from clinical studies suggests that both short-term and long-term clinical outcome of patients undergoing $\mathrm{PCI}$ is clearly associated with the inflammatory status before and after stent implantation. In particular, baseline CRP levels, more than the immediate (24-48 h) postprocedural change in CRP levels, are a reliable predictor of hard clinical end points such as death, MI or stent thrombosis. Of importance, also persistence of higher CRP 
levels at late follow-up (8-12 months) is associated with a worse clinical outcome. Mechanisms underlying the association between CRP levels and hard clinical end point are probably linked to both progression of atherosclerosis in native vessels and adverse thrombotic reactions to DES or aggressive diffuse restenosis in nonresponders to drug elution. However, it is important to consider that most studies evaluating CRP change following PCI enrolled a low number of patients and are probably underpowered to detect a significant difference in terms of clinical outcome. Moreover, most of studies evaluating the prognostic value of CRP after DES implantation are performed with first-generation DES or enrolling only a low number of patients undergoing newer-generation DES implantation. As a consequence, further studies are needed in order to investigate if CRP has a prognostic value also with more performing newer-generation DES. Moreover, in recent years bioresorbable vascular scaffolds (BVS) have been introduced into clinical practise. In particular, Absorb BVS was implanted in more than 100,000 patients. Evidence deriving from clinical studies suggests that both short-term and long-term clinical outcome of patients undergoing PCI is clearly associated with the inflammatory response before and after stent implantation. However, results deriving from large randomized clinical trials demonstrated that outcomes of Absorb BVS are inferior compared with outcomes of metallic DES [16]. In this context, it is unknown if inflammatory biomarkers may be useful to further stratify the risk of adverse events in patients undergoing BVS implantation.

\section{Financial \& competing interests disclosure}

The authors have no relevant affiliations or financial involvement with any organization or entity with a financial interest in or financial conflict with the subject matter or materials discussed in the manuscript. This includes employment, consultancies, honoraria, stock ownership or options, expert testimony, grants or patents received or pending, or royalties.

No writing assistance was utilized in the production of this manuscript.

\section{References}

1. Garg S, Serruys PW. Coronary stents: current status. J. Am. Coll. Cardiol. 56(10 Suppl.), S1-S42 (2010).

2. Montone RA, Sabato V, Sgueglia GA, Niccoli G. Inflammatory mechanisms of adverse reactions to drug-eluting stents. Curr. Vasc. Pharmacol. 11(4), 392-398 (2013).

3. Niccoli G, Montone RA, Ferrante G, Crea F. The evolving role of inflammatory biomarkers in risk assessment after stent implantation. J. Am. Coll. Cardiol. 56(22), 1783-1793 (2010).

4. Montone RA, Ferrante G, Bacà M, Niccoli G. Predictive value of C-reactive protein after drug-eluting stent implantation. Future Cardiol. 6(2), 167-179 (2010).

5. Niccoli G, Schiavino D, Belloni F et al. Pre-intervention eosinophil cationic protein serum levels predict clinical outcomes following implantation of drug-eluting stents. Eur. Heart J. 30(11), 1340-1347 (2009).

6. Park DW, Yun SC, Lee JY et al. C-reactive protein and the risk of stent thrombosis and cardiovascular events after drug-eluting stent implantation. Circulation 120(20), 1987-1995 (2009).

7. Park DW, Lee SW, Yun SC et al. A point-of-care platelet function assay and C-reactive protein for prediction of major cardiovascular events after drug-eluting stent implantation. J. Am. Coll. Cardiol. 58(25), 2630-2639 (2011).

8. Oemrawsingh RM, Cheng JM, Akkerhuis KM et al. High-sensitivity C-reactive protein predicts 10-year cardiovascular outcome after percutaneous coronary intervention. EuroIntervention 12(3), 345-351 (2016).

9. Schoos MM, Kelbæk H, Kofoed KF et al. Usefulness of preprocedure high-sensitivity C-reactive protein to predict death, recurrent myocardial infarction, and stent thrombosis according to stent type in patients with ST-segment elevation myocardial infarction randomized to bare metal or drug-eluting stenting during primary percutaneous coronary intervention. Am. J. Cardiol. 107(11), 1597-1603 (2011).

10. Niccoli G, Sgueglia GA, Latib A, Crea F, Colombo A. CACTUS Study Group. Association of baseline C-reactive protein levels with periprocedural myocardial injury in patients undergoing percutaneous bifurcation intervention: a CACTUS study subanalysis. Catheter. Cardiovasc. Interv. 83(1), E37-E44 (2014).

11. Niccoli G, Conte M, Cosentino $\mathrm{N}$ et al. Baseline C-reactive protein serum levels and in-stent restenosis pattern after $\mathrm{m}$-TOR inhibitors drug-eluting stent implantation. J. Invasive Cardiol. 23(1), 16-20 (2011).

12. Shiba M, Itaya H, Iijima R, Nakamura M. Influence of late vascular inflammation on long-term outcomes among patients undergoing implantation of drug eluting stents: role of c-reactive protein. J. Am. Heart Assoc. 5(9), e003354 (2016).

13. Hsieh IC, Chen CC, Hsieh MJ et al. Prognostic impact of 9-month high-sensitivity c-reactive protein levels on long-term clinical outcomes and in-stent restenosis in patients at 9 months after drug-eluting stent implantation. PLoS ONE 10(9), e0138512 (2015).

14. Kang WC, Il Moon C, Lee K et al. Comparison of inflammatory markers for the prediction of neointimal hyperplasia after drug-eluting stent implantation. Coron. Artery Dis. 22(8), 526-532 (2011). 
15. Lee SY, Hong MK, Mintz GS et al. Temporal course of neointimal hyperplasia following drug-eluting stent implantation: a serial follow-up optical coherence tomography analysis. Int. J. Cardiovasc. Imaging 30(6), 1003-1311 (2014).

16. Montone RA, Niccoli G, De Marco F et al. Temporal trends in adverse events after everolimus-eluting bioresorbable vascular scaffold versus everolimus-eluting metallic stent implantation: a meta-analysis of randomized controlled trials. Circulation 135(22), 2145-2154 (2017). 